\title{
The Crosstalk between Cancer Stem Cells and Microenvironment Is Critical for Solid Tumor Progression: The Significant Contribution of Extracellular Vesicles
}

\author{
Chiara Ciardiello $\mathbb{D}$, Alessandra Leone $\mathbb{D}$, and Alfredo Budillon $\mathbb{1}$ \\ Experimental Pharmacology Unit, Istituto Nazionale Tumori_IRCCS_Fondazione G. Pascale, Naples, Italy \\ Correspondence should be addressed to Chiara Ciardiello; c.ciardiello@istitutotumori.na.it
}

Received 24 July 2018; Revised 2 October 2018; Accepted 11 October 2018; Published 5 November 2018

Academic Editor: Anna T. Brini

Copyright (c) 2018 Chiara Ciardiello et al. This is an open access article distributed under the Creative Commons Attribution License, which permits unrestricted use, distribution, and reproduction in any medium, provided the original work is properly cited.

\begin{abstract}
Several evidences nowadays demonstrated the critical role of the microenvironment in regulating cancer stem cells and their involvement in tumor progression. Extracellular vesicles (EVs) are considered as one of the most effective vehicles of information among cells. Accordingly, a number of studies led to the recognition of stem cell-associated EVs as new complexes able to contribute to cell fate determination of either normal or tumor cells. In this review, we aim to highlight an existing bidirectional role of EV-mediated communication-from cancer stem cells to microenvironment and also from microenvironment to cancer stem cells-in the most widespread solid cancers as prostate, breast, lung, and colon tumors.
\end{abstract}

\section{Stemness: An Overview on Its Relevance in Cancer Development}

Cancer stem cells (CSCs) represent a critical subset of the tumor population, which has been identified more than 10 years ago, able to promote cancer initiation and progression, contributing to therapy resistance, recurrence, and metastasis [1]. CSC theory of cancer progression described them as a specific compartment of tumor cells that, similar to normal stem cells, can induce hierarchical differentiation. CSCs showed ability to self-renewal, as well as invasive capability and metastatic proficiency, so favoring tumor aggressiveness $[2,3]$. However, conflictive results have been obtained about either CSC origin or mechanisms by which CSCs serve as a critical tumor "tool" for resistance to anticancer therapy. Both an intrinsic therapy insensitivity belonging to nondividing CSC quiescent cells and resistance mechanisms activated by proliferative CSCs are hypotheses under debate. A key concept which unfolds cancer stem cell origin and dynamics in different malignancies is the "tumor plasticity," providing the idea of dynamic changes affecting cancer cells, which explain both reversible mesenchymal transitions and acquisition of stemness traits, underlying the lethal biology of metastatic dissemination and development of resistance to treatments [2-5]. Hence, CSCs themselves do not exist as a static population, and the interconversion between CSCs and non-CSCs through self-differentiation and dedifferentiation has been proposed [6]. To date, the overexpression of few stemness-related transcriptional factors has been reported as able to transform non-CSCs into CSCs in both glioblastoma [7] and colon cancer [8] models. However, in the context of cancer, dynamic changes triggering tumor plasticity are (i) the conditions the tumor is usually exposed to (i.e., hypoxia) $[9,10]$; (ii) the contribution of cell-to-cell communication exerted by EVs [11]; (iii) the tumor microenvironment (TME), composed of diverse cell types, such as mesenchymal stem cells, endothelial cells, fibroblasts, or immune cells $[3,12]$. In this regard, Quante et al. demonstrated that bone-derived myofibroblasts favored the formation of a mesenchymal stem cell niche by a differential regulation of cytokines and secretory molecules such as IL6, Wnt5 $\alpha$, and BMP4, which ultimately leads to tumor progression and recurrence [13]. Concordantly, several studies demonstrated that cancer-associated fibroblasts (CAF), resident cells commonly present in the stroma, support the stemness of CSC cells by a paracrine mechanism. Indeed, it 
has been demonstrated that by the release of cancer cellderived secretory molecules, CAFs could activate signaling functionally involved in the maintenance of stemness, as $\mathrm{Wnt} / \beta$-catenin and Notch pathways $[3,14-17]$. In return, as a feedback model, CSC influences CAF activity via activation of pathways functionally involved in cancer progression, such as Hedgehog signaling [18].

Beyond the cellular component, TME shows a noncellular component, defined as an extracellular matrix (ECM), which is composed of macromolecules such as collagens, glycoproteins, and proteoglycans as well as integrins [19, 20]. ECM, by both structure remodeling and a continuous crosstalk between tumor cells and the TME, regulates extracellular cues from the microenvironment in order to maintain CSC stemness or to promote differentiation into heterogeneous tumor phenotypes. Specifically, ECM molecules regulate CSC behaviors by modulating both cell-cell signaling and immune surveillance. For instance, tenascin-C, a protein of ECM involved in angiogenesis, invasion, and metastasis, has been recently identified as involved in the formation of the stem niche, relevant to favor lung colonization of breast cancer cells. Notably, this phenomenon seems to be dependent on the ability of tenascin- $\mathrm{C}$ to support the metastatic initiation of breast cancer cells through enhancing self-renewal pathways by increasing the expression of the regulator of stem cell signaling leucine-rich repeat containing G proteincoupled receptor 5 (LGR5) [3]. On the other hand, tenascin- $C$ itself has been shown to induce immune escape of prostate stem-like cells, by disrupting $\mathrm{T}$-cell activation [21]. Finally, tenascin-C seems to be correlated with poor prognosis in glioma patients, thus being also considered as putative CSC biomarkers for those patients [22, 23]. Both the survival of cancer cells and the formation of metastatic lesions have been recognized as deeply dependent on host microenvironment and specific organ structures, able to influence metastatic niche formation and interactions between cancer cells and local resident cells [24]. In this review, we aim to highlight an existing bidirectional role of EV-mediated communication-from cancer stem cells to microenvironment and also from microenvironment to cancer stem cells-in different solid tumors. In this context, we will describe how the CSC hypothesis provides an attractive cellular mechanism to account for the therapeutic refractoriness and dormant behavior exhibited by many solid tumors [25].

\section{Extracellular Vesicles: Different Mediators Serving Cancer Development}

Extracellular vesicles (EVs) are nowadays recognized as powerful mediators of intercellular communication in both physiological and pathological conditions. Their role in cancer development and progression has gained increasing attentions, in either hematologic or solid tumors, as broadly recapitulated in numerous reviews over the last years [26-30]. Tumor cells shed a heterogeneous set of EVs, and these spherical lipid bilayer vesicle populations differ in size, biogenesis, and molecular composition. Among the subtypes of EVs, the most studied are the exosomes $(30-100 \mathrm{~nm})$, which originate from the late endosomal trafficking machinery, gathered intracellularly into multivesicular bodies (MVBs) and shed upon MVB fusion with the plasma membrane [31]. In addition, ectosomes, apoptotic bodies (ABs), and large oncosomes (LO) represent additional subpopulations of EVs, which shared the feature to be secreted by budding from the cell plasma membrane (PM) and may express quantitatively and/ or qualitatively different types of molecular components [3235]. Actually, the ectosome category may be considered as inclusive of both $\mathrm{ABs}$ and possibly LO, which derive from apoptotic and nonapoptotic membrane blebbing processes, respectively. These two categories are both larger than $1 \mu \mathrm{m}$, and LO may even reach $10 \mu \mathrm{m}$, being also the unique population to be exclusively shed by cancer cells [36]. Microvesicles (MV) are small cell particles of heterogeneous size $(100-1000 \mathrm{~nm})$ and also PM-derived, which are extensively studied when derived from platelets and endothelial cells in relation to thrombotic disorders and diseases other than cancer [37].

One of the pioneering studies on EVs and cancer stemness was the one by Ratajczak and colleagues showing vesiclemediated horizontal transfer of mRNA and protein from embryonic stem cells as critical for the maintenance of hematopoietic stem/progenitor cell stemness and pluripotency [38]. Several years later, Stik and colleagues published in 2017 a study on mesenchymal stromal cells releasing EVs able to modulate hematopoietic stem and progenitor cell gene expressions, maintaining their survival and clonogenic potential, presumably by preventing apoptosis [39].

Thus, it is not surprising to observe that in the last 10 years, a significant amount of studies have been focused on the correlation between EVs, cancer, and stemness, as highlighted by PubMed publications (Figure 1). However, an effort by researchers to uniform the nomenclature will help advances in the field enormously. As highlighted in Figure 1, different key words (EVs vs. exosomes, ...) produced totally different outcomes despite the fact that each investigation aims to study the same topic.

\section{Extracellular Vesicles from Cancer Stem Cells (CSCs) Influence Resident Tumor Cells and Tumor Microenvironment (TME) Carrying Different Molecules}

The critical role of CSC in influencing TME has been highlighted and reviewed elsewhere [40]. The identification of new evolving communication factors in stem cell biology leads to the recognition of stem cell-associated EVs as new complexes able to contribute to cell fate determination of either normal or tumor cells. On one hand, SC-EVs could contribute to physiological activation of repair mechanisms after injury, by maintaining some key stemness features, such as self-renewal, differentiation, and maturation of damaged tissues [41]. In this regard, Tomasoni and colleagues unveiled how exosomes derived from bone marrow mesenchymal stem cells induced a horizontal transfer of insulin-like growth factor-1 mRNA, which ultimately 


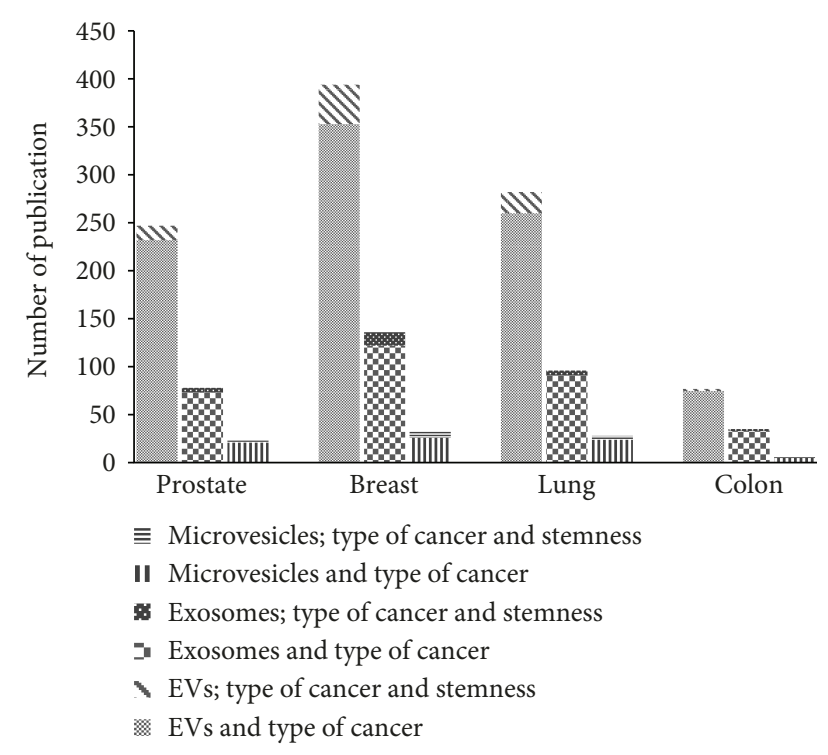

FIGURE 1: The graph shows the number of publication recorded in PubMed in the last 10 years (up to June 2018), by typing the following: 1st bar: extracellular vesicles "and" cancer (prostate, breast, lung, and colon) as well as extracellular vesicles "and" cancer "and" stemness; 2nd bar: exosomes "and" cancer (prostate, breast, lung, and colon) NOT extracellular vesicles as well as exosomes "and" cancer "and" stemness NOT extracellular vesicles; 3rd bar: microvesicles "and" cancer (prostate, breast, lung, and colon) NOT extracellular vesicles as well as microvesicles "and" cancer "and" stemness NOT extracellular vesicles.

support the repair of renal tubule after injury [42]. On the other hand, SC-EVs could influence tumor cell fate, by genetic reprogramming of resident cells and modification on TME as well as immunomodulation, which in turn could influence the tumorigenesis process [41]. Several studies have identified a number of miRNAs, such as miR-148a, miR-532-5p, miR-378, and let-7f, which, by regulating different genes involved in several multiorgan processes, could influence survival, differentiation, and immunomodulation of resident cells, including tumor cells [43-47]. In this regard, EVs derived from bone marrow mesenchymal stem cells delivered to tumor cells different miRNAs such as miR-23b and miR-21 and were able to sustain renal cell carcinoma and breast carcinoma proliferations, revealing a protumorigenic characteristic of MSC-EVs [46, 47]. Furthermore, EVs contain a large amount of proteins that could modulate several signaling pathways on resident cells. On instance, it has been demonstrated that EVs derived from mast cells acted as a shuttle for KIT proteins, which by activating its downstream pathway, leads to lung adenocarcinoma proliferation [41, 48]. Similarly, Roccaro and colleagues demonstrated that EVs from bone marrow mesenchymal stem cells transfer several cytokines, such as IL6, CCL2 (also known as MCP1), and junction plakoglobin (also known as $\gamma$-catenin), on melanoma cells, promoting tumor growth both on in vitro and in vivo models [49]. Finally, EVs carried also lipids, as diacylglycerol (DAG), sphingomyelin (SM), and ceramides, which are involved in the regulation of cell energy homeostasis as well as in crucial key pathways of tumorigenesis, such as proliferation, apoptosis, and migration $[47,50]$.

Some evidences lead to speculate that EVs, not only from stem cells but also from microenvironment, could promote, at least in part, the construction of premetastatic niches, by modulating the differentiation of the cellular component of TME $[41,51]$. In this regard, it has been reported that gastric cancer exosomes induced differentiation of mesenchymal stem cells in CAFs, by transferring activation of molecules that ultimately modulate the TGF- $\beta /$ Smad pathway [52]. In parallel, several evidences reported also a communication from cells of microenvironment on tumor cells by EV releasing, as recently reported by Shimoda et al. These authors showed that secretion of metalloproteinase-rich EVs from CAFs activates RhoA and Notch signalings, promoting cancer cell motility [53].

\section{Extracellular Vesicles: Back and Forth Messages to Build a Network among the CSC Component and TME Cells, in Different Solid Tumors}

4.1. Prostate Cancer. In the scenario of prostate cancer (PCa), studies available on CSCs (putative markers, localization within the organ, and functional studies) are still controversial, even if there are numerous evidences supporting the hierarchical model, in which a subpopulation of cells possesses the ability to initiate tumor growth and survival [54-56]. Recent studies on genetically engineered mouse models support the existence of cancer stem cells at diverse stages of tumor progression: from prostatic intraepithelial neoplasia to advanced metastatic and castration-resistant disease [57]. Maintaining CSCs in their undifferentiated stem cell state, which allows self-renewal and uninterrupted accumulation of genetic and epigenetic changes, is a condition triggered by several factors: one of the most studied in PCa is hypoxia [58]. From one hand, hypoxia has been identified as a promoting factor of metabolic changes, oncogene activation, and epithelial mesenchymal transition, resistant to chemo- and radiotherapy [59]; from the other hand, it has been also shown as able to affect EV-mediated communication [60]. Exosomes secreted by hypoxic cells were enriched in both HSP90 and HSP70 and expressed higher levels of annexin II compared to exosomes secreted by cells in normoxic conditions [61]. HSP90 has been described as abundantly secreted by organoids with cancer stem cell-like properties [62]; annexin II is also implicated in the metastatic process [63], and its expression in numerous cancers correlates with resistance to treatment, binding to the bone marrow, histological grade and type, TNM stage, and shortened overall survival, as discussed in a recent review [64]. Exosomes secreted by hypoxic condition-exposed $\mathrm{PCa}$ cell lines were able to either enhance the ability of naïve PCa cells to form prostasphere or promote the cancer-associated phenotype in prostateassociated CAF [61]. Indeed, exosomes have been shown to contain signaling molecules as TGF- $\beta 2$, TNF1 $\alpha$, IL6, Akt, ILK1, and $\beta$-catenin primarily associated with the 

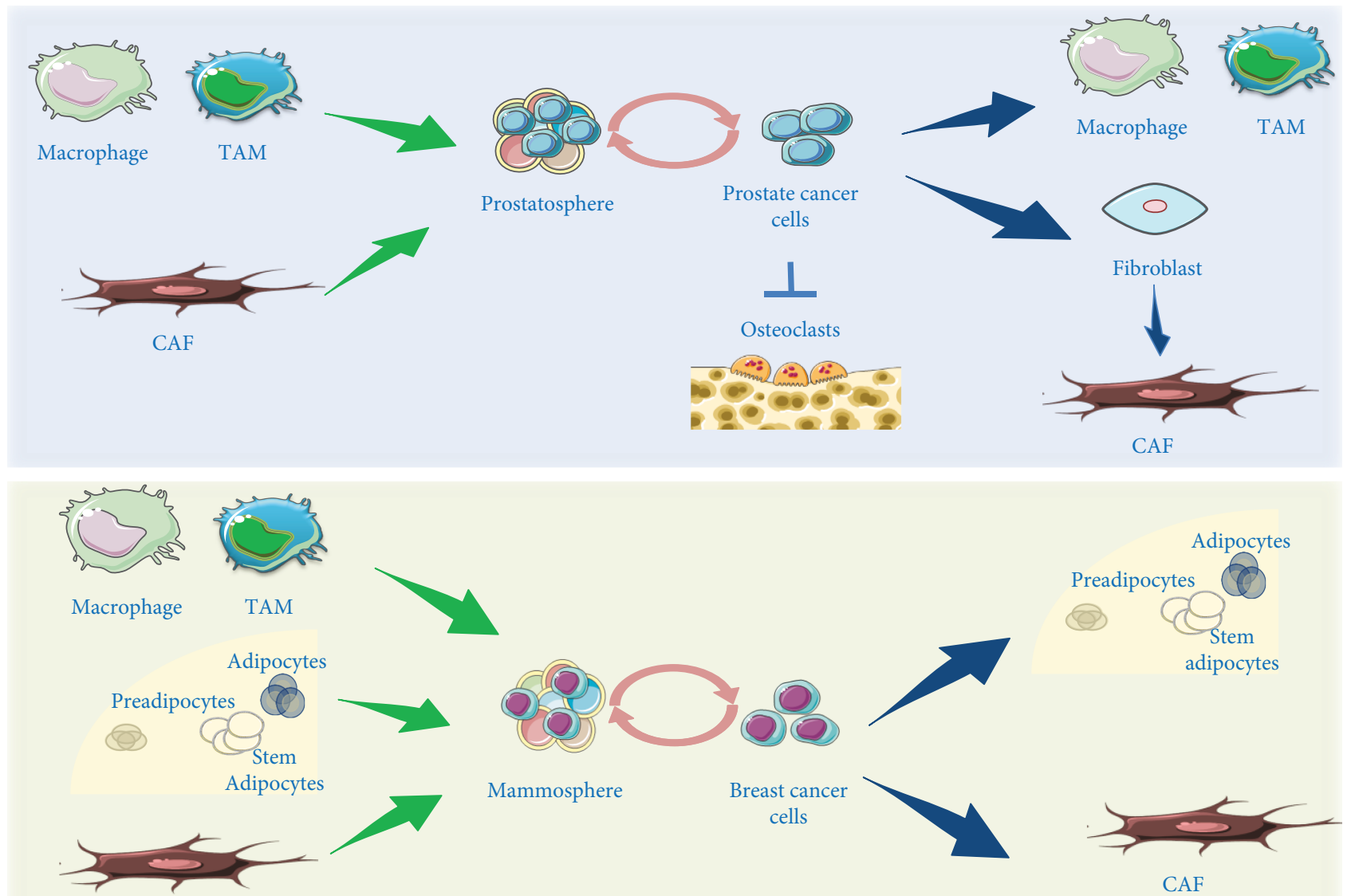

CAF

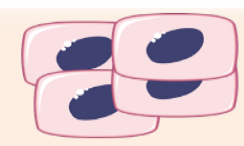

Endothelial cells

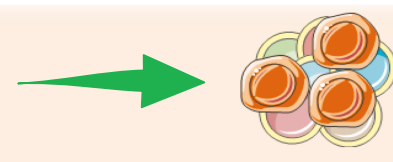

Colon sphere

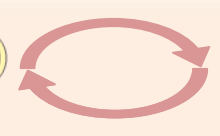

Colon cancer cells
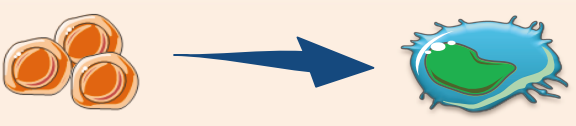

TAM

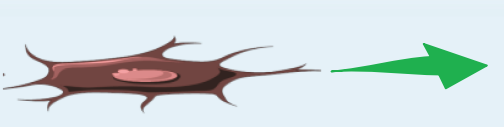

CAF

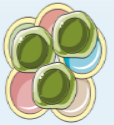

Lung sphere

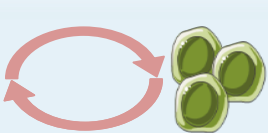

Lung cancer cells

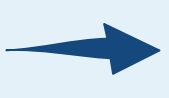

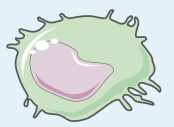

Macrophage

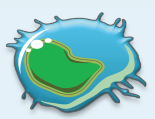

TAM

FIGURE 2: Specific interaction between microenvironment/metastatic niche components (i.e., fibroblasts, adipocytes, and macrophages) and organ-specific tumor cells through EVs, described in detail in the text. Tumor spheres are representative of a CSC-enriched tumor compartment, while tumor cells indicated a differentiated compartment. Green arrows indicated EV-mediated signals from microenvironment to tumor, blue arrows indicated EV-mediated signals from tumor to microenvironment, and light pink arrows indicated autocrine signaling mediated by EVs from tumor cells to the tumor sphere and vice versa.

remodeling of the epithelial adherens junction pathway and stemness feature development [61].

The back and forth communication between PCa and TME to support stemness-related pathways is mostly studied referring to CAF, the key recipient of messages carried by EVs from PCa cell [65] macrophages and bone component (Figure 2). In detail, it has been recently shown that LO shed

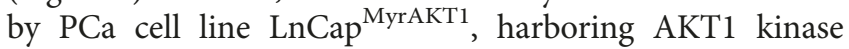

activity, were internalized by human normal prostate fibroblasts, inducing their reprogramming through the activation of stromal Myc [66], a proto-oncogene implicated in cancer initiation, maintenance, and stemness in different models $[67,68]$. However, also miRNAs, carried by PCa CSCderived EVs, have been shown to target fibroblast, affecting their proliferation, differentiation, and migration. Sánchez and colleagues compared exosomal miRNA shed from the 
"bulk component" versus CSC-enriched prostatosphere, both obtained from patient-derived primary cell cultures. They found hsa-miR-100-5p as the higher expressed in exosomes from both origin, compared to the other miRNAs [69]. miR-122 and let7b were both differentially expressed in CSC exosomes compared to bulk-derived exosomes [69], although their implications as stemness promoters are actually controversial $[70,71]$. Some of the miRNAs coming out from that study are able to affect fibroblast properties as migration [69]. Conversely, it has been also reported that stromal fibroblast-derived miR-409 exported by EVs was able to induce activation of oncogenic, proliferative, EMT, and stemness programs of adjacent tumor epithelia in vivo; specifically, SOX2 and Nanog were both elevated in miR409-expressing fibroblast [72]. An interesting study by Huang and colleagues unveiled the reciprocal network between CSC and macrophages, another major component of TME. In detail, the authors observed that the autophagyrelated gene 7 (ATG7) facilitated the transcription of Oct4 via $\beta$-catenin, promoting CSC characteristics in prostate cancer, including self-renewal, tumor initiation, and drug resistance. In addition, also CSCs remodeled their specific niche by educating monocytes/macrophages towards tumor-associated macrophages (TAMs), and the CSCeducated TAMs reciprocally promoted the stem-like properties of CSCs as well as progression and ADT resistance of prostate cancer via interleukin 6 (IL6)/STAT3 [73]. Although in the latter study EVs were not mentioned at all, ILs exporting through EVs have been described [74].

Despite the fact that the bone is the preferential site of metastasis for breast and prostate tumors [75], only few studies explored the intercellular communication between PCa and both osteoblast and osteoclast. In detail, Karlsson and colleagues showed that exosomes isolated from the murine PCa cell line TRAMP-C1 dramatically decreased the fusion and differentiation of osteoclast precursors to mature multinucleated osteoclasts [76]. A clear decrease in the expression of established markers for osteoclast fusion and differentiation, including DC-STAMP, TRAP, cathepsin K, and MMP-9 was observed upon exposure to PCa-derived exosomes [76]. Inder and colleagues worked on EVs derived from the PC3 PCa cell line demonstrating that PC3-derived EVs were internalized by both osteoclast precursors and primary human osteoblast, inducing, respectively, osteoclastogenesis and proliferation [77].

4.2. Breast Cancer. Tumor initiation, therapeutic resistance, relapse, and metastasis have been associated to the concept of stemness and plasticity also in breast cancer [78]. It has been observed that in vitro models of breast cancer cells enriched in stemness features and grown as mammosphere showed a high expression of Rab27A (a member of RAS oncogene family), able to increase the exocytosis of EVs, compared to adherent breast cancer cell models [79]. Several studies focused on the effort to identify molecular cargos in EVs derived from breast cancer stem cells. miR-155 has been identified as enriched in exosomes isolated from breast CSCs, leading to EMT-associated chemoresistance [80]. miR-140, miR-29a, and miR-21 have been found to be enriched in exosomes derived from basal-like ductal carcinoma in situ (DCIS) stem-like cells. miR-9, upregulated in various breast cancer cell lines and identified as prometastatic miRNA, is delivered by exosomes and is able to affect the properties of human breast fibroblasts, enhancing the switch to CAF phenotype [81]. More recently, not only miR-9 but also miR-221 have been both shown to enable breast cancer cells to generate spheroids with stem cell-like characteristics [82]. A growing body of evidences reported EVs released from diverse cells belonging to the TME and targeting breast cancer stem cells. Among the variegated set of stromal cell types, numerous investigators have focused their work on bone marrow-derived cells, endothelial cells, fibroblasts, mesenchymal stem cell ability to influence tumor growth, and progression (Figure 2). In particular, on one hand, the ability of transformed fibroblasts to induce stemness markers in cancer cells (c-Myc/ miR-34a circuitry deregulation, SOX2 upregulation, ...) has been recently pointed out by Bono and colleagues [83]. In depth, miRNAs are often carried by exosomes as it has been nicely reviewed elsewhere [84]. Exosomes released by CAF have been shown to shuttle miR-21, -378 e, and -143 and make breast cancer cell lines more efficient to form mammospheres, upregulating stemness-related transcriptional factors such as Oct3-4, Nanog, and SOX2 and promoting EMT via ZEB1 induction [85]. Already in 2003, other cell types belonging to TME gained attention as promoters of cancer aggressive features: adipocytes and adipose tissue-derived mesenchymal stem cells might contribute to a stem cell-like phenotype in breast cancer [86]. Despite the fact that this latest study by Iyengar and colleagues did not mention EVs, it suggested that "adipokines" were able to induce the expression of prooncogenic factors such as beta-catenin and CDK6 as a result of a reduction in the gene expression of their inhibitors in breast cancer recipient cells [86]. More recently, Baglio and colleagues set up a protocol for isolating exosomes released by both early passage adipose stem cell (ASC) and bone marrow MSCs (BMSC) and observed a selective export of miRNA in exosomes, not always reflecting the whole miRNA set of the cell of origin, thus suggesting a selective packaging process through EVs. miR-486-5p, miR-10a-5p, miR-10b-5p, miR-191-5p, and miR-222-3p were found to be the most abundant miRNAs in ASC exosomes, while miR-143-3p, miR-10b-5p, miR486-5p, miR-22-3p, and miR-21-5p in BMSC exosomes [87]. Intriguingly, another study in 2015 showed that exosomes secreted from preadipocytes have been identified as important components of the cancer stem cell niche, significantly contributing, upon internalization by early-stage breast cancer cells, to mammosphere formation and growth [88]. On the opposite side, miR-503-3p, isolated from human adipose stem cell- (ASC-) derived exosomes, suppressed initiation and progression of CSCs, suppressing tumor sphere formation and decreasing the expression of pluripotency genes [89]. However, in order to highlight the reciprocity between breast cancer cells and adipocytes in communication through EVs, it is worth to mention that EVs shed by MDA MB-231 human breast cancer cells promote hallmark features of myofibroblastic 
differentiation and proangiogenic behavior in adiposederived stem cells (ASCs) [90].

4.3. Lung Cancer. Lung cancer is one of the most common types of cancer, representing the leading cause of cancerrelated deaths worldwide [91]. New lung cancer diagnoses increased 14\%, and mortality related to lung cancer accounted for approximately 1 of every 4 cancer deaths in 2016 [91]. Two main histological subtypes of lung cancer have been described: small cell (SCLC) and NSCLC, the latter being the most frequent (close to $80-85 \%$ of all lung cancers) and aggressive ( $>5$-year survival rate of 10\%) [92]. Compared to other types of cancers, lung CSC markers have been poorly defined and explored since lung cancer is considered one of the most genotypic and histologically complex tumors [93]. Singh and colleagues showed that the signaling axis EGFR/Src/Akt is able to positively modulate SOX2 expression and self-renewal of stem-like side population cells in NSCLC [94]. More recently, NF- $\kappa \mathrm{B}$ inhibition has been shown to be sufficient to prevent the EMT and to induce apoptosis in lung CSCs, defined as $\mathrm{CD} 166^{+} \mathrm{CD} 44^{+}$, $\mathrm{CD} 66^{+} \mathrm{EpCAM}^{+}$cells [95]. Leprieur and colleagues described a Sonic Hedgehog ( $\mathrm{SH}$ ) membrane-bound fulllength form, characterizing the CSCs compartment in human NSCLC, which has been observed to be secreted by CSCs, in vitro [96]. In vivo, analyzing 48 fresh human surgical samples, compared to healthy controls, the authors confirmed the in vitro observations suggesting paracrine and autocrine functions for $\mathrm{SH}$ protein, being responsible for CSC maintenance, tumor proliferation, and resistance to chemotherapy [96]. The CSC population in NSCLC has been recently defined also by the overexpression of the long noncoding DGCR5, which is able to regulate the expression of CD44 by modulating miR-330-5p [97]. The interplay and crosstalk between CSCs and TME, particularly CAF, have been shown to be relevant for lung cancer progression (Figure 2). Recently, Plou and colleagues demonstrated that modulation of collagen concentration and the amount of TGF- $\beta$ in the microenvironment can regulate the plasticity of lung cancer cells, supporting the formation of tumor clusters, commonly considered enriched of putative tumorinitiating cells [98]. Furthermore, several studies demonstrated that CAFs promote lung tumorigenesis by activating a paracrine crosstalk with cancer cells and more importantly, with lung CSCs [99]. Indeed, Chen and colleagues identified the paracrine network by which the primary component of the NSCLC microenvironment, CAFs, enriches CSCs through dedifferentiation and reacquisition of stem cell-like properties. Specifically, they found that IGF1R signaling activation in cancer cells in the presence of CAFs expressing IGF-II can induce Nanog expression and promote stemness. Interestingly, the authors pointed out that this paracrine signaling predicts overall and relapse-free survival in stage I NSCLC patients [100].

Recently, several studies have highlighted the role of EVs containing CSC-priming factors as main agents to promote TME/CSC communication. To date, the literature available on EVs and lung cancer is mainly focused on the exosome population: a significant effort has been employed to define the exosomal miRNA content as diagnostic biomarkers for liquid biopsy, and, in parallel, EVcontaining miRNAs have been demonstrated to play pleiotropic functions in regulating tumor malignancy [101]. A list of the main candidates has been published in a recent review [102]. However, what is immediately noticeable among these studies is either the variability in the sources of exosomes employed (mainly plasma and also sera or pleural effusions) or the higher variability in the methods to collect exosomes, thus affecting the reproducibility of these data sets.

A recent study by $\mathrm{Hsu}$ and colleagues observed that EVs shed by the human lung cancer cell line CL1-5 exposed to hypoxic conditions were enriched in miR103a compared to EVs collected from the same cell line exposed to normoxic conditions [103]. They also showed that this EV-carried miR-103a was able to affect macrophage phenotype, inducing a tumor-promoting behavior via PTEN modulation [103]. Similarly, it has been reported that EVs such as exosomes secreted by $\mathrm{H} 460$ and A549 lung cancer cells modulate the tumor microenvironment, by influencing tumor cell migration. Mechanistically, the authors unveiled that restoration of LKB1, by targeting multiple critical signaling pathways, including AMPK/mTOR, p53, and PTEN/AKT, is able to inhibit exosomal secretion of migration-suppressing microRNAs (miRNAs), such as miR-125a, miR-126, and let7b [104].

4.4. Colon Cancer. Colorectal cancer (CRC) is nowadays considered as cause of approximately $10 \%$ of cancerrelated mortality in western countries. As Kuipers and colleagues highlighted in 2015, diverse factors determined the rise in CRC incidence over the past 60 years, such as increasing ageing population, unfavorable modern dietary habits, and an increase in risk factors, such as smoking, low physical exercise, and obesity [105]. Feng and colleagues showed that, similar to other cancers and as mentioned above, Rab27A overexpression is correlated to increased sphere formation efficiency (SFE) and elevated secretion of VEGF and TGF- $\beta$ from HT29 CRC cells [106]. They also found a correlation between a higher p65 level and Rab27A in the colon cancer sphere, demonstrating that NF- $\kappa \mathrm{B}$ signaling promotes various colon cancer stem cell properties via an amplified paracrine mechanism regulated by the higher Rab27A level [106]. To date, exosomes from CRC cells may export molecules in a selective manner, as mentioned above for other models: in detail, Cha and colleagues showed that the KRAS status of CRC cells can affect the type of miRNAs enriched in exosomes [107]. Considering the biological context in which CRC develops, it is worth to mention that there is a well-established link between CRC and chronic inflammation, which has been recently revised [108]. In detail, among the immune system cells, recent studies provide strong evidences that TAMs might facilitate CRC growth $[109,110]$. Specifically, it has been shown that TAMs can stimulate CRC growth by altering extracellular matrix remodeling, tumor metabolism, angiogenesis, and the TME [110]. However, there are no studies focusing on the crosstalk between TAM and CRC; only a recent report 
TABLE 1: Overview of biological interactions between tumor microenvironment and extracellular vesicles in solid cancer tumors.

\begin{tabular}{|c|c|c|c|c|c|}
\hline Model & TME components & EV type & Cargo & Molecular targets & Ref. \\
\hline \multirow{4}{*}{ Prostate cancer } & \multirow{2}{*}{$\mathrm{NAF} / \mathrm{CAF}$} & LO & $\mathrm{AKT}$ & Myc activation & {$[66]$} \\
\hline & & Exosomes & $\operatorname{miR}-409$ & SOX and Nanog activation & {$[72]$} \\
\hline & Macrophages & n.d. & ATG7 or IL6/STAT3 & $\beta$-Catenin and Oct 4 activation & {$[73]$} \\
\hline & Osteoclasts & Exosomes & n.d. & $\begin{array}{l}\text { DC-STAMP, TRAP, cathepsin K, and } \\
\text { MMP9 inhibition }\end{array}$ & {$[76]$} \\
\hline \multirow{3}{*}{ Breast cancer } & \multirow{2}{*}{$\mathrm{NAF} / \mathrm{CAF}$} & \multirow{2}{*}{ Exosomes } & miR-9 & EFEMP1, COL1A1, and MMP1 & {$[81]$} \\
\hline & & & $\operatorname{miR}-21,-378 \mathrm{e}$, and -143 & Oct $3 / 4$, SOX2, and Nanog activation & {$[85]$} \\
\hline & Preadipocytes & Exosomes & miR-149 & SOX signaling & {$[88]$} \\
\hline \multirow{2}{*}{ Lung cancer } & Macrophages & Exosomes & miR-103a & PTEN modulation & [103] \\
\hline & n.d. & n.d. & miR-125a, miR-126, and let7b & LKB1, AMPK/mTOR, p53, and PTEN/AKT & {$[104]$} \\
\hline \multirow{3}{*}{ Colorectal cancer } & $\begin{array}{l}\text { Mesenchymal } \\
\text { stromal cells }\end{array}$ & Exosomes & n.d. & Vacuolar $\mathrm{H}^{+}$-ATPase protein & [112] \\
\hline & Macrophages & n.d. & n.d. & IL33/prostaglandin E2 signaling & {$[113]$} \\
\hline & n.d. & Exosomes & miR-196b-5p & STAT3 signaling & [114] \\
\hline
\end{tabular}

unveiled that tumor-derived exosomes induce $\mathrm{PD}^{+}$macrophage population in human gastric cancer, promoting disease progression [111]. Exosomes from CRC have been indeed shown to directly induce the activation of mesenchymal stromal cells (MSC), isolated from colonic mucosa [112]. In detail, Lugini and colleagues showed that CRC exosomes are able to induce changes in the morphology of MSC accompanied by higher proliferation, migration, and invasion, formation of large $3 \mathrm{D}$ spheroids. In addition, they observed that colon cancer-derived MSCs, isolated from colon adenocarcinoma cell masses, fully recapitulate the changes observed in normal colonic MSCs exposed to CRC exosomes [112]. To support the TME in colon cancer, IL33, expressed by both cancer cells and endothelial cells, has been recently shown to stimulate CRC sphere formation and prevent chemotherapy-induced tumor apoptosis [113]. In detail, IL33 recruited macrophages into the cancer microenvironment and stimulated them to produce prostaglandin E2, which supported CRC stemness and tumor growth. In a recent study by Ren and colleagues, miR-196b-5p has been shown to promote either stemness or chemoresistance of CRC cells to 5-fluorouracil via targeting negative regulators of the STAT3 signaling pathway. Authors also found miR196b-5p highly enriched in the serum exosomes of patients with CRC, compared to the healthy control subjects [114].

\section{Conclusions}

Although several pathways have been identified as mainly involved in maintaining a stem-supportive microenvironment in cancer development, the EVs role as mediators of stem signaling still needs to be deeply understood. In summary, what is known from recent literature is that on one hand, EVs derived from CSCs could influence tumor cell fate, by genetic reprogramming of resident cells and influencing the TME including immune cells (Figure 2 and Table 1); on the other hand, a specific component of the microenvironment (i.e., fibroblasts, adipocytes, and macrophages) may be able to modify the tumor niche by
EV shedding in a diverse type of solid tumors (Figure 2 and Table 1). The characterization of the exact role of these different EVs and of their mRNA, miRNA, DNA, and/or protein cargos could help in the definition of novel tumor biomarkers as well as therapeutic targets.

\section{Conflicts of Interest}

The authors declare that they have no competing interests.

\section{Authors' Contributions}

CC and AL worked on the concept and drafted and edited the manuscript. $\mathrm{AB}$ took care of critical revision of the manuscript and final approval. All authors read and approved the final manuscript. Alessandra Leone and Alfredo Budillon contributed equally to this work.

\section{Acknowledgments}

The authors are grateful for financial support from "Associazione Italiana Ricerca sul Cancro" (AIRC). This study was funded by AIRC (3-year Fellowship AIRCFIRC for Italy 19586 to CC) and by "Ricerca Corrente" funds (Italian Ministry of Health).

\section{References}

[1] S. M. Tu, S. H. Lin, and C. J. Logothetis, "Stem-cell origin of metastasis and heterogeneity in solid tumours," The Lancet Oncology, vol. 3, no. 8, pp. 508-513, 2002.

[2] A. Leone, M. S. Roca, C. Ciardiello, S. Costantini, and A. Budillon, "Oxidative stress gene expression profile correlates with cancer patient poor prognosis: identification of crucial pathways might select novel therapeutic approaches," Oxidative Medicine and Cellular Longevity, vol. 2017, Article ID 2597581, 8 pages, 2017.

[3] A. Z. Ayob and T. S. Ramasamy, "Cancer stem cells as key drivers of tumour progression," Journal of Biomedical Science, vol. 25, no. 1, p. 20, 2018. 
[4] D. Jia, M. K. Jolly, P. Kulkarni, and H. Levine, "Phenotypic plasticity and cell fate decisions in cancer: insights from dynamical systems theory," Cancers, vol. 9, no. 12, 2017.

[5] V. Plaks, N. Kong, and Z. Werb, "The cancer stem cell niche: how essential is the niche in regulating stemness of tumor cells?,” Cell Stem Cell, vol. 16, no. 3, pp. 225-238, 2015.

[6] Z. Sun, L. Wang, L. Dong, and X. Wang, "Emerging role of exosome signalling in maintaining cancer stem cell dynamic equilibrium," Journal of Cellular and Molecular Medicine, vol. 22, no. 8, pp. 3719-3728, 2018.

[7] I. Olmez, W. Shen, H. McDonald, and B. Ozpolat, "Dedifferentiation of patient-derived glioblastoma multiforme cell lines results in a cancer stem cell-like state with mitogen-independent growth," Journal of Cellular and Molecular Medicine, vol. 19, no. 6, pp. 1262-1272, 2015.

[8] N. Oshima, Y. Yamada, S. Nagayama et al., "Induction of cancer stem cell properties in colon cancer cells by defined factors," PLoS One, vol. 9, no. 7, article e101735, 2014.

[9] C. Peitzsch, R. Perrin, R. P. Hill, A. Dubrovska, and I. Kurth, "Hypoxia as a biomarker for radioresistant cancer stem cells," International Journal of Radiation Biology, vol. 90, no. 8, pp. 636-652, 2014.

[10] J. Guo, B. Wang, Z. Fu, J. Wei, and W. Lu, "Hypoxic microenvironment induces EMT and upgrades stem-like properties of gastric cancer cells," Technology in Cancer Research \& Treatment, vol. 15, no. 1, pp. 60-68, 2016.

[11] T. T. Liao and M. H. Yang, "Revisiting epithelialmesenchymal transition in cancer metastasis: the connection between epithelial plasticity and stemness," Molecular Oncology, vol. 11, no. 7, pp. 792-804, 2017.

[12] J. Ye, D. Wu, P. Wu, Z. Chen, and J. Huang, "The cancer stem cell niche: cross talk between cancer stem cells and their microenvironment," Tumour Biology, vol. 35, no. 5, pp. 3945-3951, 2014.

[13] M. Quante, S. P. Tu, H. Tomita et al., "Bone marrow-derived myofibroblasts contribute to the mesenchymal stem cell niche and promote tumor growth," Cancer Cell, vol. 19, no. 2, pp. 257-272, 2011.

[14] L. J. A. C. Hawinkels, M. Paauwe, H. W. Verspaget et al., "Interaction with colon cancer cells hyperactivates TGF- $\beta$ signaling in cancer-associated fibroblasts," Oncogene, vol. 33, no. 1, pp. 97-107, 2014.

[15] L. Vermeulen, F. de Sousa E Melo, M. van der Heijden et al., "Wnt activity defines colon cancer stem cells and is regulated by the microenvironment," Nature Cell Biology, vol. 12, no. 5, pp. 468-476, 2010.

[16] E. Y. T. Lau, J. Lo, B. Y. L. Cheng et al., "Cancer-associated fibroblasts regulate tumor-initiating cell plasticity in hepatocellular carcinoma through c-Met/FRA1/HEY1 signaling," Cell Reports, vol. 15, no. 6, pp. 1175-1189, 2016.

[17] Y. Yu, C. H. Xiao, L. D. Tan, Q. S. Wang, X. Q. Li, and Y. M. Feng, "Cancer-associated fibroblasts induce epithelialmesenchymal transition of breast cancer cells through paracrine TGF- $\beta$ signalling," British Journal of Cancer, vol. 110, no. 3, pp. 724-732, 2014.

[18] G. Valenti, H. M. Quinn, G. J. J. E. Heynen et al., "Cancer stem cells regulate cancer-associated fibroblasts via activation of Hedgehog signaling in mammary gland tumors," Cancer Research, vol. 77, no. 8, pp. 2134-2147, 2017.
[19] J. M. Muncie and V. M. Weaver, "The physical and biochemical properties of the extracellular matrix regulate cell fate," Current Topics in Developmental Biology, vol. 130, pp. 1-37, 2018.

[20] T. Alkasalias, L. Moyano-Galceran, M. Arsenian-Henriksson, and K. Lehti, "Fibroblasts in the tumor microenvironment: shield or spear?," International Journal of Molecular Sciences, vol. 19, no. 5, article 1532, 2018.

[21] E. Jachetti, S. Caputo, S. Mazzoleni et al., "Tenascin-C protects cancer stem-like cells from immune surveillance by arresting T-cell activation," Cancer Research, vol. 75, no. 10, pp. 2095-2108, 2015.

[22] N. Brosicke and A. Faissner, "Role of tenascins in the ECM of gliomas," Cell Adhesion \& Migration, vol. 9, no. 1-2, pp. 131140, 2015.

[23] R. Chiquet-Ehrismann, G. Orend, M. Chiquet, R. P. Tucker, and K. S. Midwood, "Tenascins in stem cell niches," Matrix Biology, vol. 37, pp. 112-123, 2014.

[24] W. Chen, A. D. Hoffmann, H. Liu, and X. Liu, "Organotropism: new insights into molecular mechanisms of breast cancer metastasis," npj Precision Oncology, vol. 2, no. 1, p. 4, 2018.

[25] J. E. Visvader and G. J. Lindeman, "Cancer stem cells in solid tumours: accumulating evidence and unresolved questions," Nature Reviews Cancer, vol. 8, no. 10, pp. 755-768, 2008.

[26] C. Ciardiello, L. Cavallini, C. Spinelli et al., "Focus on extracellular vesicles: new frontiers of cell-to-cell communication in cancer," International Journal of Molecular Sciences, vol. 17, no. 2, p. 175, 2016.

[27] V. R. Minciacchi, M. R. Freeman, and D. Di Vizio, "Extracellular vesicles in cancer: exosomes, microvesicles and the emerging role of large oncosomes," Seminars in Cell \& Developmental Biology, vol. 40, pp. 41-51, 2015.

[28] B. Dos Anjos Pultz, F. A. C. da Luz, S. S. Faria et al., "The multifaceted role of extracellular vesicles in metastasis: priming the soil for seeding," International Journal of Cancer, vol. 140, no. 11, pp. 2397-2407, 2017.

[29] H. Zhao, A. Achreja, E. Iessi et al., "The key role of extracellular vesicles in the metastatic process," Biochimica et Biophysica Acta (BBA) - Reviews on Cancer, vol. 1869, no. 1, pp. 6477, 2018.

[30] Z. Litwinska, K. Luczkowska, and B. Machalinski, "Extracellular vesicles in hematological malignancies," Leukemia \& Lymphoma, pp. 1-8, 2018.

[31] B. T. Pan, K. Teng, C. Wu, M. Adam, and R. M. Johnstone, "Electron microscopic evidence for externalization of the transferrin receptor in vesicular form in sheep reticulocytes," The Journal of Cell Biology, vol. 101, no. 3, pp. 942-948, 1985.

[32] E. Lázaro-Ibáñez, A. Sanz-Garcia, T. Visakorpi et al., "Different gDNA content in the subpopulations of prostate cancer extracellular vesicles: apoptotic bodies, microvesicles, and exosomes," Prostate, vol. 74, no. 14, pp. 1379-1390, 2014.

[33] T. R. Lunavat, L. Cheng, D. K. Kim et al., "Small RNA deep sequencing discriminates subsets of extracellular vesicles released by melanoma cells - evidence of unique microRNA cargos," RNA Biology, vol. 12, no. 8, pp. 810-823, 2015.

[34] J. P. Tosar, F. Gambaro, J. Sanguinetti, B. Bonilla, K. W. Witwer, and A. Cayota, "Assessment of small RNA sorting into different extracellular fractions revealed by highthroughput sequencing of breast cell lines," Nucleic Acids Research, vol. 43, no. 11, pp. 5601-5616, 2015. 
[35] R. Xu, D. W. Greening, A. Rai, H. Ji, and R. J. Simpson, "Highly-purified exosomes and shed microvesicles isolated from the human colon cancer cell line LIM1863 by sequential centrifugal ultrafiltration are biochemically and functionally distinct," Methods, vol. 87, pp. 11-25, 2015.

[36] V. R. Minciacchi, S. You, C. Spinelli et al., "Large oncosomes contain distinct protein cargo and represent a separate functional class of tumor-derived extracellular vesicles," Oncotarget, vol. 6, no. 13, pp. 11327-11341, 2015.

[37] F. Santilli, M. Marchisio, P. Lanuti, A. Boccatonda, S. Miscia, and G. Davì, "Microparticles as new markers of cardiovascular risk in diabetes and beyond," Thrombosis and Haemostasis, vol. 116, no. 2, pp. 220-234, 2016.

[38] J. Ratajczak, K. Miekus, M. Kucia et al., "Embryonic stem cellderived microvesicles reprogram hematopoietic progenitors: evidence for horizontal transfer of mRNA and protein delivery," Leukemia, vol. 20, no. 5, pp. 847-856, 2006.

[39] G. Stik, S. Crequit, L. Petit et al., "Extracellular vesicles of stromal origin target and support hematopoietic stem and progenitor cells," The Journal of Cell Biology, vol. 216, no. 7, pp. 2217-2230, 2017.

[40] S. Chen and E. H. Huang, "The colon cancer stem cell microenvironment holds keys to future cancer therapy," Journal of Gastrointestinal Surgery, vol. 18, no. 5, pp. 1040-1048, 2014.

[41] M. Nawaz, F. Fatima, K. C. Vallabhaneni et al., "Extracellular vesicles: evolving factors in stem cell biology," Stem Cells International, vol. 2016, Article ID 1073140, 17 pages, 2016.

[42] S. Tomasoni, L. Longaretti, C. Rota et al., "Transfer of growth factor receptor mRNA via exosomes unravels the regenerative effect of mesenchymal stem cells," Stem Cells and Development, vol. 22, no. 5, pp. 772-780, 2013.

[43] L. De Luca, S. Trino, I. Laurenzana et al., "Mesenchymal stem cell derived extracellular vesicles: a role in hematopoietic transplantation?," International Journal of Molecular Sciences, vol. 18, no. 5, 2017.

[44] S. Bruno, M. C. Deregibus, and G. Camussi, "The secretome of mesenchymal stromal cells: role of extracellular vesicles in immunomodulation," Immunology Letters, vol. 168, no. 2, pp. 154-158, 2015.

[45] S. R. Baglio, D. M. Pegtel, and N. Baldini, "Mesenchymal stem cell secreted vesicles provide novel opportunities in (stem) cell-free therapy," Frontiers in Physiology, vol. 3, p. 359, 2012.

[46] M. Ono, N. Kosaka, N. Tominaga et al., "Exosomes from bone marrow mesenchymal stem cells contain a microRNA that promotes dormancy in metastatic breast cancer cells," Science Signaling, vol. 7, no. 332, article ra63, 2014.

[47] K. C. Vallabhaneni, P. Penfornis, S. Dhule et al., "Extracellular vesicles from bone marrow mesenchymal stem/stromal cells transport tumor regulatory microRNA, proteins, and metabolites," Oncotarget, vol. 6, no. 7, pp. 4953-4967, 2015.

[48] H. Xiao, C. Lässer, G. Shelke et al., "Mast cell exosomes promote lung adenocarcinoma cell proliferation - role of KITstem cell factor signaling," Cell Communication and Signaling, vol. 12, no. 1, p. 64, 2014.

[49] A. M. Roccaro, A. Sacco, P. Maiso et al., "BM mesenchymal stromal cell-derived exosomes facilitate multiple myeloma progression," The Journal of Clinical Investigation, vol. 123, no. 4, pp. 1542-1555, 2013.

[50] R. S. Lindoso, F. Collino, and A. Vieyra, "Extracellular vesicles as regulators of tumor fate: crosstalk among cancer stem cells, tumor cells and mesenchymal stem cells," Stem Cell Investigation, vol. 4, no. 9, p. 75, 2017.

[51] C. Grange, M. Tapparo, F. Collino et al., "Microvesicles released from human renal cancer stem cells stimulate angiogenesis and formation of lung premetastatic niche," Cancer Research, vol. 71, no. 15, pp. 5346-5356, 2011.

[52] J. Gu, H. Qian, L. Shen et al., "Gastric cancer exosomes trigger differentiation of umbilical cord derived mesenchymal stem cells to carcinoma-associated fibroblasts through TGF- $\beta$ / Smad pathway," PLoS One, vol. 7, no. 12, article e52465, 2012.

[53] M. Shimoda, S. Principe, H. W. Jackson et al., "Loss of the Timp gene family is sufficient for the acquisition of the CAF-like cell state," Nature Cell Biology, vol. 16, no. 9, pp. 889-901, 2014.

[54] N. Guzmán-Ramírez, M. Völler, A. Wetterwald et al., "In vitro propagation and characterization of neoplastic stem/ progenitor-like cells from human prostate cancer tissue," Prostate, vol. 69, no. 15, pp. 1683-1693, 2009.

[55] A. T. Collins, P. A. Berry, C. Hyde, M. J. Stower, and N. J. Maitland, "Prospective identification of tumorigenic prostate cancer stem cells," Cancer Research, vol. 65, no. 23, pp. 10946-10951, 2005.

[56] D. Jaworska, W. Krol, and E. Szliszka, "Prostate cancer stem cells: research advances," International Journal of Molecular Sciences, vol. 16, no. 11, pp. 27433-27449, 2015.

[57] M. Shibata and M. M. Shen, "Stem cells in geneticallyengineered mouse models of prostate cancer," EndocrineRelated Cancer, vol. 22, no. 6, pp. T199-T208, 2015.

[58] Z. Yun and Q. Lin, "Hypoxia and regulation of cancer cell stemness," Advances in Experimental Medicine and Biology, vol. 772, pp. 41-53, 2014.

[59] E. Paolicchi, F. Gemignani, M. Krstic-Demonacos, S. Dedhar, L. Mutti, and S. Landi, "Targeting hypoxic response for cancer therapy," Oncotarget, vol. 7, no. 12, pp. 13464-13478, 2016.

[60] M. C. Lowry and L. O'Driscoll, "Can hi-jacking hypoxia inhibit extracellular vesicles in cancer?," Drug Discovery Today, vol. 23, no. 6, pp. 1267-1273, 2018.

[61] A. Ramteke, H. Ting, C. Agarwal et al., "Exosomes secreted under hypoxia enhance invasiveness and stemness of prostate cancer cells by targeting adherens junction molecules," Molecular Carcinogenesis, vol. 54, no. 7, pp. 554$565,2015$.

[62] T. Eguchi, C. Sogawa, Y. Okusha et al., "Organoids with cancer stem cell-like properties secrete exosomes and HSP90 in a 3D nanoenvironment," PLoS One, vol. 13, no. 2, article e0191109, 2018.

[63] C. Y. Wang and C. F. Lin, "Annexin A2: its molecular regulation and cellular expression in cancer development," Disease Markers, vol. 2014, Article ID 308976, 10 pages, 2014.

[64] M. V. Christensen, C. K. Høgdall, K. M. Jochumsen, and E. V. S. Høgdall, "Annexin A2 and cancer: a systematic review," International Journal of Oncology, vol. 52, no. 1, pp. 5-18, 2018.

[65] S. Kato, A. Goodman, V. Walavalkar, D. A. Barkauskas, A. Sharabi, and R. Kurzrock, "Hyperprogressors after immunotherapy: analysis of genomic alterations associated with accelerated growth rate," Clinical Cancer Research, vol. 23, no. 15, pp. 4242-4250, 2017.

[66] V. R. Minciacchi, C. Spinelli, M. Reis-Sobreiro et al., "Myc mediates large oncosome-induced fibroblast reprogramming 
in prostate cancer," Cancer Research, vol. 77, no. 9, pp. 23062317, 2017.

[67] C. C. Cheng, L. H. Shi, X. J. Wang et al., "STAT3/Oct4/c-Myc signal circuit for regulating stemness-mediated doxorubicin resistance of triple-negative breast cancer cells and inhibitory effects of WP1066," International Journal of Oncology, vol. 53, no. 1, pp. 339-348, 2018.

[68] H. Li, Y. Jin, Y. Hu et al., "The PLGF/c-Myc/miR-19a axis promotes metastasis and stemness in gallbladder cancer," Cancer Science, vol. 109, no. 5, pp. 1532-1544, 2018.

[69] C. A. Sánchez, E. I. Andahur, R. Valenzuela et al., "Exosomes from bulk and stem cells from human prostate cancer have a differential microRNA content that contributes cooperatively over local and pre-metastatic niche," Oncotarget, vol. 7, no. 4, pp. 3993-4008, 2016.

[70] J. Zhu, S. Wang, W. Zhang et al., "Screening key microRNAs for castration-resistant prostate cancer based on miRNA/ mRNA functional synergistic network," Oncotarget, vol. 6, no. 41, pp. 43819-43830, 2015.

[71] C. H. Hung, T. H. Hu, S. N. Lu et al., "Circulating microRNAs as biomarkers for diagnosis of early hepatocellular carcinoma associated with hepatitis B virus," International Journal of Cancer, vol. 138, no. 3, pp. 714-720, 2016.

[72] S. Josson, M. Gururajan, S. Y. Sung et al., "Stromal fibroblastderived miR-409 promotes epithelial-to-mesenchymal transition and prostate tumorigenesis," Oncogene, vol. 34, no. 21, pp. 2690-2699, 2015.

[73] H. Huang, C. Wang, F. Liu et al., "Reciprocal network between cancer stem-like cells and macrophages facilitates the progression and androgen deprivation therapy resistance of prostate cancer," Clinical Cancer Research, vol. 24, no. 18, pp. 4612-4626, 2018.

[74] A. MacKenzie, H. L. Wilson, E. Kiss-Toth, S. K. Dower, R. A. North, and A. Surprenant, "Rapid secretion of interleukin-1 $\beta$ by microvesicle shedding," Immunity, vol. 15, no. 5, pp. 825835, 2001.

[75] G. Battafarano, M. Rossi, F. Marampon, and A. del Fattore, "Cellular and molecular mediators of bone metastatic lesions," International Journal of Molecular Sciences, vol. 19, no. 6, 2018.

[76] T. Karlsson, M. Lundholm, A. Widmark, and E. Persson, "Tumor cell-derived exosomes from the prostate cancer cell line TRAMP-C1 impair osteoclast formation and differentiation," PLoS One, vol. 11, no. 11, article e0166284, 2016.

[77] K. L. Inder, J. E. Ruelcke, L. Petelin et al., "Cavin-1/PTRF alters prostate cancer cell-derived extracellular vesicle content and internalization to attenuate extracellular vesiclemediated osteoclastogenesis and osteoblast proliferation," Journal of Extracellular Vesicles, vol. 3, no. 1, 2014.

[78] V. Angeloni, P. Tiberio, V. Appierto, and M. G. Daidone, "Implications of stemness-related signaling pathways in breast cancer response to therapy," Seminars in Cancer Biology, vol. 31, pp. 43-51, 2015.

[79] F. Feng, J. Zhang, X. Fan et al., "Downregulation of Rab27A contributes to metformin-induced suppression of breast cancer stem cells," Oncology Letters, vol. 14, no. 3, pp. 2947-2953, 2017.

[80] J. C. Santos, N. . S. Lima, L. O. Sarian, A. Matheu, M. L. Ribeiro, and S. F. M. Derchain, "Exosome-mediated breast cancer chemoresistance via miR-155 transfer," Scientific Reports, vol. 8, no. 1, p. 829, 2018.
[81] S. Baroni, S. Romero-Cordoba, I. Plantamura et al., "Exosome-mediated delivery of miR-9 induces cancer-associated fibroblast-like properties in human breast fibroblasts," Cell Death \& Disease, vol. 7, no. 7, article e2312, 2016.

[82] C. W. Cheng, J. C. Yu, Y. H. Hsieh et al., "Increased cellular levels of microRNA-9 and microRNA-221 correlate with cancer stemness and predict poor outcome in human breast cancer," Cellular Physiology and Biochemistry, vol. 48, no. 5, pp. 2205-2218, 2018.

[83] B. Bono, P. Ostano, M. Peritore et al., "Cells with stemness features are generated from in vitro transformed human fibroblasts," Scientific Reports, vol. 8, no. 1, p. 13838, 2018.

[84] N. Tran, "Cancer exosomes as miRNA factories," Trends in Cancer, vol. 2, no. 7, pp. 329-331, 2016.

[85] E. Donnarumma, D. Fiore, M. Nappa et al., "Cancer-associated fibroblasts release exosomal microRNAs that dictate an aggressive phenotype in breast cancer," Oncotarget, vol. 8, no. 12, pp. 19592-19608, 2017.

[86] P. Iyengar, T. P. Combs, S. J. Shah et al., “Adipocyte-secreted factors synergistically promote mammary tumorigenesis through induction of anti-apoptotic transcriptional programs and proto-oncogene stabilization," Oncogene, vol. 22, no. 41, pp. 6408-6423, 2003.

[87] S. R. Baglio, K. Rooijers, D. Koppers-Lalic et al., "Human bone marrow- and adipose-mesenchymal stem cells secrete exosomes enriched in distinctive miRNA and tRNA species," Stem Cell Research \& Therapy, vol. 6, no. 1, p. 127, 2015.

[88] R. Gernapudi, Y. Yao, Y. Zhang et al., "Targeting exosomes from preadipocytes inhibits preadipocyte to cancer stem cell signaling in early-stage breast cancer," Breast Cancer Research and Treatment, vol. 150, no. 3, pp. 685-695, 2015.

[89] M. Seo, S. M. Kim, E. Y. Woo et al., "Stemness-attenuating miR-503-3p as a paracrine factor to regulate growth of cancer stem cells," Stem Cells International, vol. 2018, Article ID 4851949, 10 pages, 2018.

[90] Y. H. Song, C. Warncke, S. J. Choi et al., "Breast cancerderived extracellular vesicles stimulate myofibroblast differentiation and pro-angiogenic behavior of adipose stem cells," Matrix Biology, vol. 60-61, pp. 190-205, 2017.

[91] L. A. Torre, R. L. Siegel, and A. Jemal, "Lung cancer statistics," Advances in Experimental Medicine and Biology, vol. 893, pp. 1-19, 2016.

[92] M. Zhang, G. Li, Y. Wang et al., "PD-L1 expression in lung cancer and its correlation with driver mutations: a meta-analysis," Scientific Reports, vol. 7, no. 1, p. 10255, 2017.

[93] A. Giangreco, K. R. Groot, and S. M. Janes, "Lung cancer and lung stem cells: strange bedfellows?," American Journal of Respiratory and Critical Care Medicine, vol. 175, no. 6, pp. 547-553, 2007.

[94] S. Singh, J. Trevino, N. Bora-Singhal et al., "EGFR/Src/Akt signaling modulates SOX2 expression and self-renewal of stem-like side-population cells in non-small cell lung cancer," Molecular Cancer, vol. 11, no. 1, p. 73, 2012.

[95] N. Zakaria, N. Mohd Yusoff, Z. Zakaria, D. Widera, and B. H. Yahaya, "Inhibition of NF- $\kappa \mathrm{B}$ signaling reduces the stemness characteristics of lung cancer stem cells," Frontiers in Oncology, vol. 8, p. 166, 2018.

[96] E. G. Leprieur, B. Tolani, H. Li et al., "Membrane-bound fulllength Sonic Hedgehog identifies cancer stem cells in human non-small cell lung cancer," Oncotarget, vol. 8, no. 61, pp. 103744-103757, 2017. 
[97] R. Wang, H. X. Dong, J. Zeng, J. Pan, and X. Y. Jin, "LncRNA DGCR5 contributes to CSC-like properties via modulating miR-330-5p/CD44 in NSCLC," Journal of Cellular Physiology, vol. 233, no. 9, pp. 7447-7456, 2018.

[98] J. Plou, Y. Juste-Lanas, V. Olivares, C. del Amo, C. Borau, and J. M. García-Aznar, "From individual to collective 3D cancer dissemination: roles of collagen concentration and TGF- $\beta$," Scientific Reports, vol. 8, no. 1, p. 12723, 2018.

[99] C. Alguacil-Núñez, I. Ferrer-Ortiz, E. García-Verdú, P. López- Pirez, I. M. Llorente-Cortijo, and B. Sainz Jr, "Current perspectives on the crosstalk between lung cancer stem cells and cancer-associated fibroblasts," Critical Reviews in Oncology/Hematology, vol. 125, pp. 102-110, 2018.

[100] W. J. Chen, C. C. Ho, Y. L. Chang et al., "Cancer-associated fibroblasts regulate the plasticity of lung cancer stemness via paracrine signalling," Nature Communications, vol. 5, no. 1, p. 3472, 2014.

[101] Y. Fujita, N. Kosaka, J. Araya, K. Kuwano, and T. Ochiya, "Extracellular vesicles in lung microenvironment and pathogenesis," Trends in Molecular Medicine, vol. 21, no. 9, pp. 533-542, 2015.

[102] C. Masaoutis, C. Mihailidou, G. Tsourouflis, and S. Theocharis, "Exosomes in lung cancer diagnosis and treatment. From the translating research into future clinical practice," Biochimie, vol. 151, pp. 27-36, 2018.

[103] Y. L. Hsu, J. Y. Hung, W. A. Chang et al., "Hypoxic lungcancer-derived extracellular vesicle microRNA-103a increases the oncogenic effects of macrophages by targeting PTEN," Molecular Therapy, vol. 26, no. 2, pp. 568-581, 2018.

[104] C. Zhang, X. Xiao, M. Chen, H. Aldharee, Y. Chen, and W. Long, "Liver kinase B1 restoration promotes exosome secretion and motility of lung cancer cells," Oncology Reports, vol. 39, no. 1, pp. 376-382, 2018.

[105] E. J. Kuipers, W. M. Grady, D. Lieberman et al., "Colorectal cancer," Nature Reviews Disease Primers, vol. 1, article 15066, 2015.

[106] F. Feng, Y. Jiang, H. Lu et al., "Rab27A mediated by NF- $\kappa B$ promotes the stemness of colon cancer cells via upregulation of cytokine secretion," Oncotarget, vol. 7, no. 39, pp. 63342-63351, 2016.

[107] D. J. Cha, J. L. Franklin, Y. Dou et al., "KRAS-dependent sorting of miRNA to exosomes," eLife, vol. 4, article e07197, 2015.

[108] Y. Yang, C. Xu, D. Wu et al., " $\gamma \delta$ T cells: crosstalk between microbiota, chronic inflammation, and colorectal cancer," Frontiers in Immunology, vol. 9, p. 1483, 2018.

[109] J. K. Colby, J. Jaoude, F. Liu, and I. Shureiqi, “Oxygenated lipid signaling in tumor-associated macrophages-focus on colon cancer," Cancer Metastasis Reviews, vol. 37, no. 2-3, pp. 289-315, 2018.

[110] X. Zhong, B. Chen, and Z. Yang, "The role of tumorassociated macrophages in colorectal carcinoma progression," Cellular Physiology and Biochemistry, vol. 45, no. 1, pp. 356-365, 2018.

[111] F. Wang, B. Li, Y. Wei et al., "Tumor-derived exosomes induce $\mathrm{PD}^{+}$macrophage population in human gastric cancer that promotes disease progression," Oncogene, vol. 7, no. 5, p. 41, 2018.

[112] L. Lugini, M. Valtieri, C. Federici et al., "Exosomes from human colorectal cancer induce a tumor-like behavior in colonic mesenchymal stromal cells," Oncotarget, vol. 7, no. 31, pp. 50086-50098, 2016.
[113] M. Fang, Y. Li, K. Huang et al., "IL33 promotes colon cancer cell stemness via JNK activation and macrophage recruitment," Cancer Research, vol. 77, no. 10, pp. 2735-2745, 2017.

[114] D. Ren, B. Lin, X. Zhang et al., "Maintenance of cancer stemness by miR-196b-5p contributes to chemoresistance of colorectal cancer cells via activating STAT3 signaling pathway," Oncotarget, vol. 8, no. 30, pp. 49807-49823, 2017. 


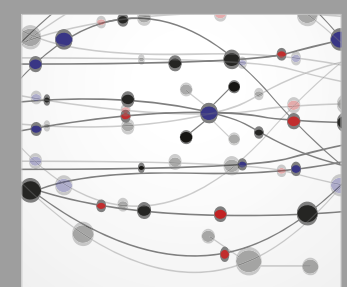

The Scientific World Journal
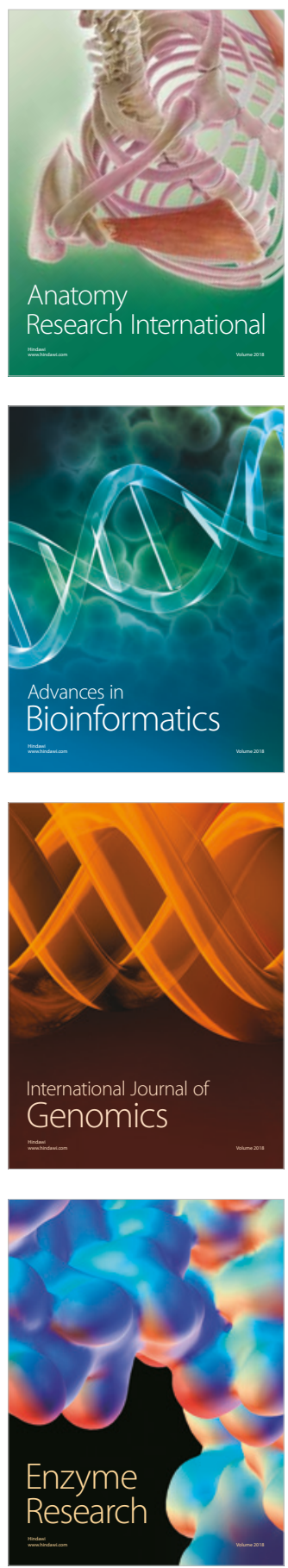
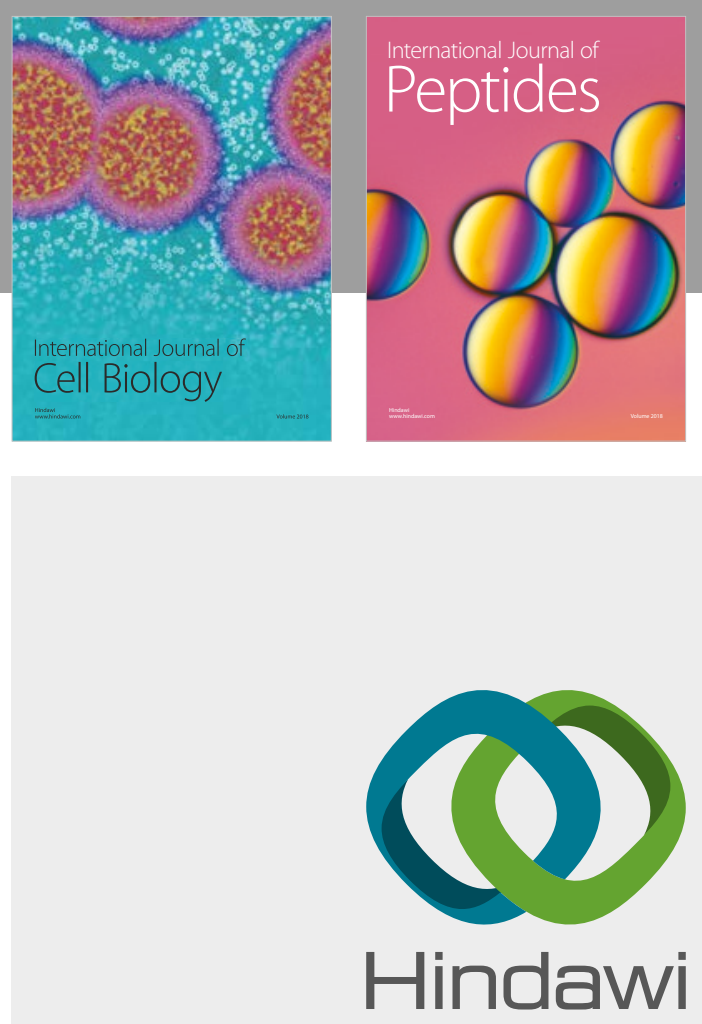

Submit your manuscripts at

www.hindawi.com
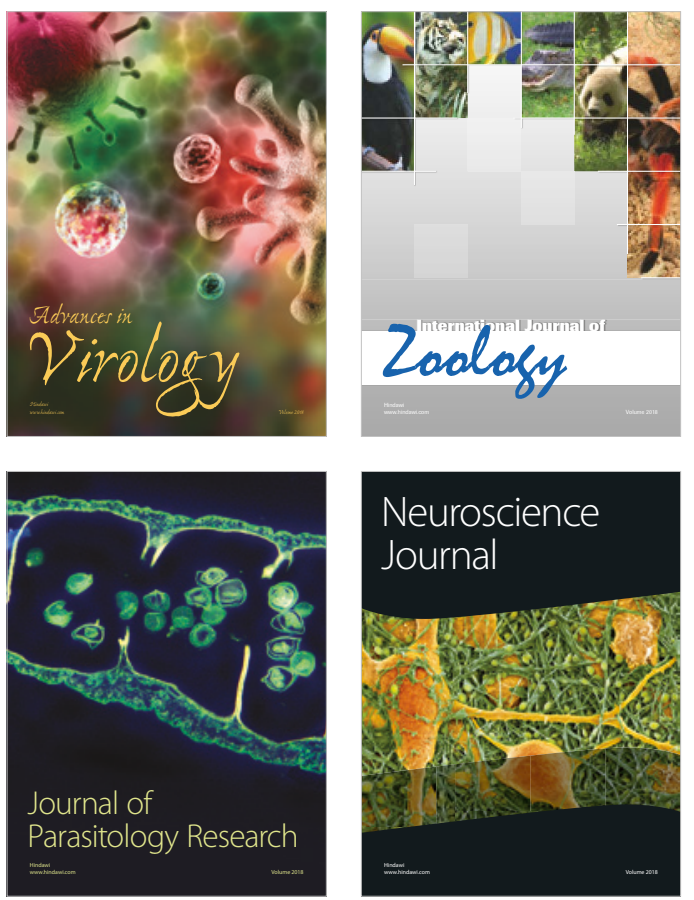
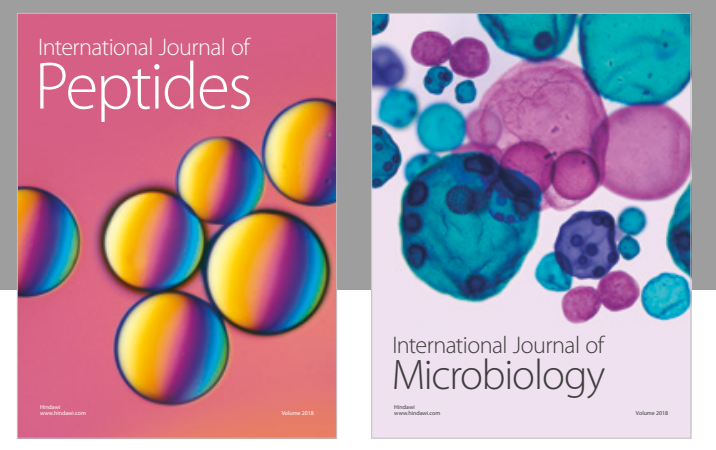

nternational Journal of Microbiology
Journal of
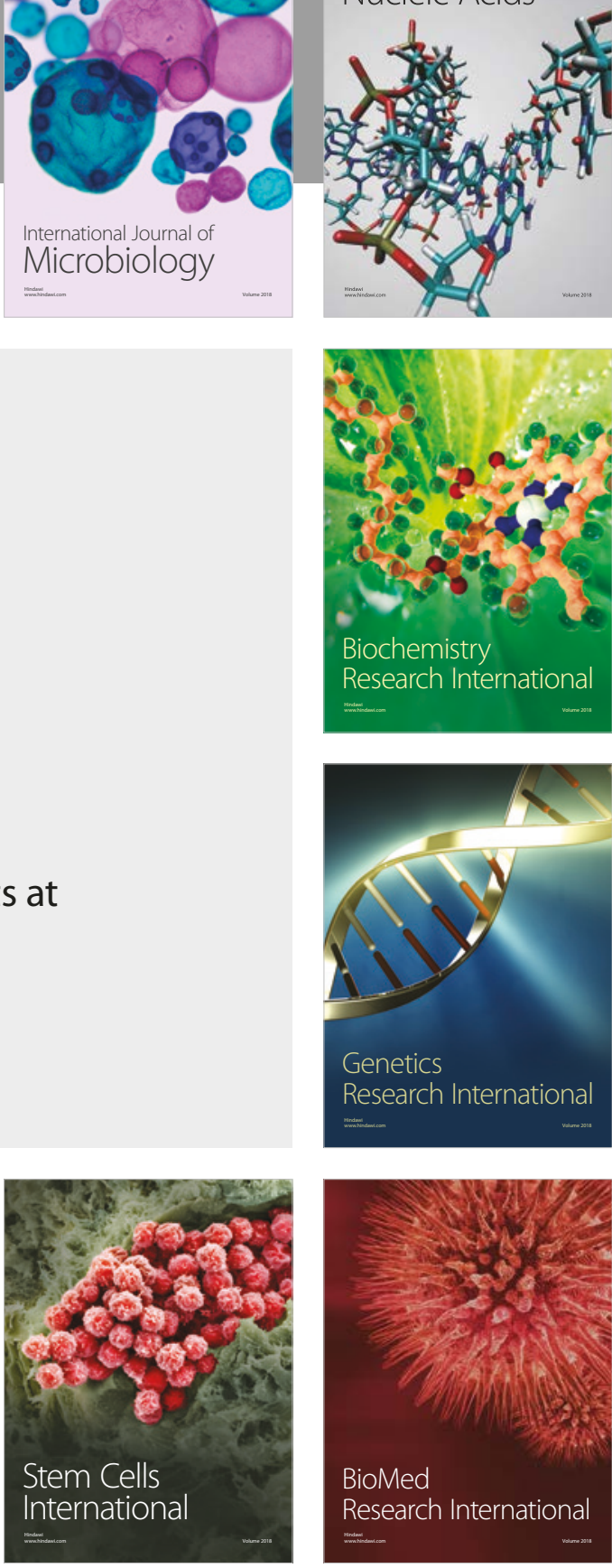
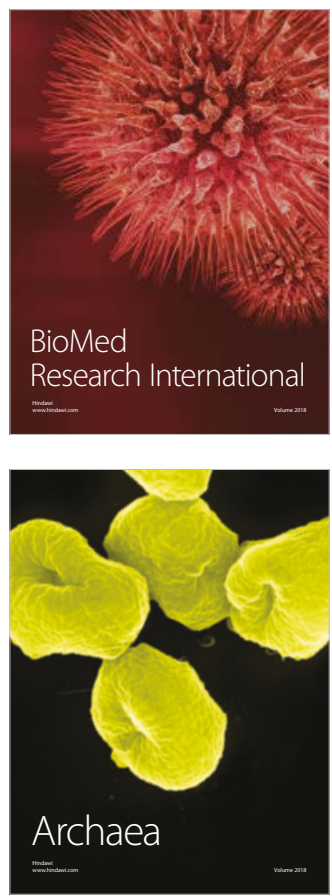